\title{
Introduction to the Special Issue on Data-Intensive Computing in Smart Microgrids
}

\author{
Herodotos Herodotou
}

check for updates

Citation: Herodotou, $\mathrm{H}$. Introduction to the Special Issue on Data-Intensive Computing in Smart Microgrids. Energies 2021, 14, 2704. https://doi.org/10.3390/ en14092704

Received: 28 April 2021

Accepted: 6 May 2021

Published: 9 May 2021

Publisher's Note: MDPI stays neutral with regard to jurisdictional claims in published maps and institutional affiliations.

Copyright: (C) 2021 by the author. Licensee MDPI, Basel, Switzerland. This article is an open access article distributed under the terms and conditions of the Creative Commons Attribution (CC BY) license (https:/ / creativecommons.org/licenses/by/ $4.0 /)$.
Department of Electrical Engineering, Computer Engineering and Informatics, Cyprus University of Technology, Limassol 3036, Cyprus; herodotos.herodotou@cut.ac.cy

Microgrids have recently emerged as the building block of a smart grid combining distributed renewable energy sources, energy storage devices, and load management in order to improve power system reliability, enhance sustainable development, and reduce carbon emissions. At the same time, the rapid advancements in sensor and metering technologies, wireless and network communication, as well as cloud and fog computing are leading to the collection and accumulation of large amounts of data (e.g., device status data, energy generation data, consumption data).

The application of big data analysis techniques (e.g., forecasting, classification, clustering) on such data can optimize the power generation and operation in real time by accurately predicting electricity demands, discovering electricity consumption patterns, and developing dynamic pricing mechanisms. The efficient and intelligent analysis of the data will enable smart microgrids to detect and restore from failures quickly, respond to electricity demand swiftly, supply more reliable and economical energy, and enable customers to have more control over their energy use. Overall, data-intensive analytics can provide effective and efficient decision support for all of the producers, operators, customers, and regulators in smart microgrids in order to achieve holistic smart energy management, including energy generation, transmission, distribution, and demand-side management.

We are pleased to present the Special Issue of Energies (ISSN 1996-1073) entitled "DataIntensive Computing in Smart Microgrids", which contains an assortment of relevant research contributions. This Special Issue received a total of 14 manuscript submissions, out of which nine were selected for publication after a rigorous peer-review process. In the following, we provide a brief overview of the eight research articles and one review manuscript featured in this Special Issue.

In the article by Ahmad et al. [1], a new machine learning-based methodology is proposed for short-term electricity load forecasting. The methodology consists of three main steps: (i) feature selection using a combination of an XGBoost and a decision tree feature selector, (ii) redundancy removal using recursive feature elimination, and (iii) forecasting using support vector machine (SVM) and extreme learning machine (ELM). The hyper-parameters of SVM are tuned with the grid search algorithm while the hyperparameters of ELM are tuned with a genetic algorithm.

Masood et al. [2] investigate three narrowband power line communication (NB-PLC) channel modeling techniques over medium voltage networks for advanced metering infrastructure. A transmission line theory model expresses the network as a two-port network to examine the characteristics of sending and receiving NB-PLC signals. A multipath signal propagation model incorporates the effect of multipath signals to determine the NB-PLC transfer function. Finally, a Simulink model considers the values of the network to examine the characteristics of NB-PLC signals.

In the article by Bukhsh et al. [3], the energy demand of a fog (micro) data center is evaluated by calculating the amount of energy required to run computing devices based on the number of requests as well as the amount of energy needed for cooling down the devices. The fog-based model can be used to ensure real-time energy management service 
provision for a green community. Various scenarios are simulated for fulfilling the energy demand from the utility and/or the use of renewable energy resources.

Ayub et al. [4] propose a framework for short- and medium-term electricity forecasting using a combination of deep learning and supervised machine learning techniques. First, Random Forest and XGBoost are used for identifying the most important features. Next, recursive feature elimination is performed to remove any redundant features. Finally, load forecasting is performed with support vector machines (SVMs) and a hybrid of convolutional neural networks (CNNs) and gated recurrent units (GRUs). Two metaheuristic algorithms, namely, grey wolf optimization and earth worm optimization, are utilized to tune the hyper-parameters of SVMs and CNNs-GRUs, respectively.

Aslam et al. [5] address the problem of electricity theft detection in smart grids by proposing a modeling technique that combines deep learning (namely, long short-term memory (LSTM) neural networks and UNet) with ensemble learning (namely, adaptive boosting). In addition, the authors propose a new class balancing mechanism based on the interquartile minority oversampling technique to overcome the high-dimensional imbalanced data that are often used in these studies.

Qureshi et al. [6] propose an energy-efficient resource allocation strategy for real-time data-intensive smart systems that periodically offload computational and data-intensive loads to the cloud. The proposed strategy utilizes a mathematical model and a group of algorithms for minimizing the data files transfer overhead to computing resources by selecting appropriate pairs of computing and storage resources.

In the article by Ullah et al. [7], an energy optimization strategy is proposed to minimize operation cost and carbon emissions in a smart grid integrated with renewable energy sources. First, probability density functions are suggested to predict the behavior of wind and solar energy sources. To overcome uncertainty in power produced by wind and solar sources, demand response programs (DRPs) are proposed with the involvement of residential, commercial, and industrial consumers. Finally, incentive-based payments are introduced as price offered packages for executing the DRPs.

Ali et al. [8] propose a new optimization-based energy management framework that adapts consumer power usage patterns using real-time pricing signals and generation from utility and photovoltaic-battery systems in order to minimize electricity cost, reduce carbon emission, and mitigate peak power consumption. A hybrid genetic ant colony optimization algorithm is proposed to solve the complete scheduling model with or without photovoltaic and photovoltaic-battery systems.

Ullah et al. [9] provide a comprehensive review of various automatic generation control (AGC) models in diverse configurations of electric power systems. An in-depth analysis of various control methods used to mitigate the AGC issues is provided. The application of fast-acting energy storage devices, high voltage direct current interconnections, and flexible AC transmission systems devices in the AGC systems are also investigated. Furthermore, AGC systems employed in different renewable energy generation systems as well as in different configurations of microgrids/smart grids are discussed and compared.

Finally, we would like to thank all the authors who have contributed their papers for this Special Issue. We are sincerely grateful to the reviewers who contributed to assembling such a high-quality Special Issue. We are also indebted to the Energies editors, editorial office, and the publishing and production teams for their assistance in the preparation and publication of this Special Issue.

Conflicts of Interest: The author declares no conflict of interests.

\section{References}

1. Ahmad, W.; Ayub, N.; Ali, T.; Irfan, M.; Awais, M.; Shiraz, M.; Glowacz, A. Towards Short Term Electricity Load Forecasting Using Improved Support Vector Machine and Extreme Learning Machine. Energies 2020, 13, 2907. [CrossRef]

2. Masood, B.; Khan, M.A.; Baig, S.; Song, G.; Rehman, A.U.; Rehman, S.U.; Asif, R.M.; Rasheed, M.B. Investigation of Deterministic, Statistical and Parametric NB-PLC Channel Modeling Techniques for Advanced Metering Infrastructure. Energies 2020, 13, 3098. [CrossRef] 
3. Bukhsh, R.; Javed, M.U.; Fatima, A.; Javaid, N.; Shafiq, M.; Choi, J.-G. Cost Efficient Real Time Electricity Management Services for Green Community Using Fog. Energies 2020, 13, 3164. [CrossRef]

4. Ayub, N.; Irfan, M.; Awais, M.; Ali, U.; Ali, T.; Hamdi, M.; Alghamdi, A.; Muhammad, F. Big Data Analytics for Short and Medium-Term Electricity Load Forecasting Using an AI Techniques Ensembler. Energies 2020, 13, 5193. [CrossRef]

5. Aslam, Z.; Javaid, N.; Ahmad, A.; Ahmed, A.; Gulfam, S.M. A Combined Deep Learning and Ensemble Learning Methodology to Avoid Electricity Theft in Smart Grids. Energies 2020, 13, 5599. [CrossRef]

6. Qureshi, M.S.; Qureshi, M.B.; Fayaz, M.; Zakarya, M.; Aslam, S.; Shah, A. Time and Cost Efficient Cloud Resource Allocation for Real-Time Data-Intensive Smart Systems. Energies 2020, 13, 5706. [CrossRef]

7. Ullah, K.; Ali, S.; Khan, T.A.; Khan, I.; Jan, S.; Shah, I.A.; Hafeez, G. An Optimal Energy Optimization Strategy for Smart Grid Integrated with Renewable Energy Sources and Demand Response Programs. Energies 2020, 13, 5718. [CrossRef]

8. Ali, S.; Khan, I.; Jan, S.; Hafeez, G. An Optimization Based Power Usage Scheduling Strategy Using Photovoltaic-Battery System for Demand-Side Management in Smart Grid. Energies 2021, 14, 2201. [CrossRef]

9. Ullah, K.; Basit, A.; Ullah, Z.; Aslam, S.; Herodotou, H. Automatic Generation Control Strategies in Conventional and Modern Power Systems: A Comprehensive Overview. Energies 2021, 14, 2376. [CrossRef] 